\title{
Movement of the tympanic membrane causing pulsatile tinnitus
}

\author{
Supaporn Srirompotong, Somchai Srirompotong \\ Department of Otorhinolaryngology, Faculty of Medicine, Khon Kaen University, Khon Kaen 40002, \\ Thailand
}

\begin{abstract}
Background: Movement of the tympanic membrane can be a cause of pulsatile tinnitus. There are many etiologies that cause movement of the tympanic membrane, including otogenic pneumocephalus. This is an uncommon presentation after head injury that may be missed as the diagnosis of the primary cause of the symptoms.

Objective: To report a rare case of pulsatile tinnitus because of otogenic pneumocephalus.

Methods: Case report from chart review with relevant investigations and treatment.

Results: We describe the very interesting case of a patient who presented with pulsatile tinnitus because of otogenic pneumocephalus. On examination, we found a moving tympanic membrane. A CT scan revealed pneumocephalus in the right temporoparietal region that was continuous with the adjacent mastoid air cells. There was a transverse fracture through the mastoid process. A meticulous mastoidectomy with unroofing of the epitympanum was performed with widening of the aditus ad antrum. The mastoid cavity was closed without fat obliteration. After the operation, the pulsatile tinnitus ceased completely.

Conclusion: Mastoidectomy and unroofing the epitympanum without fat obliteration is a very simple and effective option for the treatment of pulsatile tinnitus because of pneumocephalus.
\end{abstract}

Keywords: Moving tympanic membrane, pneumocephalus, pulsatile tinnitus

Pulsatile tinnitus is a type of ear noise that is perceived as a rhythmic pulsing that is often synchronous with the heartbeat. Pulsatile tinnitus usually originates from vascular structures because of either increased blood flow or lumen stenosis, atherosclerotic carotid or subclavian artery disease, arterial, venous, or arteriovenous malformations, fistulae, or dissection and paragangliomas [1]. The other causes include chronic inflammation and/or infection of the middle ear and middle ear fluid.

A moving tympanic membrane can be the cause of rhythmic tinnitus. There are many etiologies that cause movement of the tympanic membrane, such as vascular tumors in the middle ear, a patulous Eustachian tube, and otogenic pneumocephalus. We present the case of a patient with pulsatile tinnitus. On examination, we found a moving tympanic membrane. The symptoms completely resolved after mastoidectomy. This is an uncommon presentation after head injury that we may miss as the primary

Correspondence to: Supaporn Srirompotong, Department of Otorhinolaryngology, Faculty of Medicine, Khon Kaen University, Khon Kaen 40002, Thailand. E-mail: ssrirompotong@ gmail.com cause of symptoms.

Publication of this case report was approved by the human ethics committee of Khon Kaen University (Institutional Review Board Number: IRB00001189) following the criteria of the Declaration of Helsinki in accordance with standards of Good Clinical Practice provided by the International Conference on Harmonisation.

\section{Case report}

A 19-year-old woman presented with a 6-month history of pulsatile tinnitus in her right ear that initially began after craniotomy surgery. Seven months previously, she had a head injury and was diagnosed as having an epidural hematoma in the right frontotemporoparietal area that required a craniotomy for clot removal. Subsequently she developed pulsatile tinnitus in her right ear that was synchronous with her pulse. The symptom was present continuously. The tinnitus was severe enough to disturb her daily activities and sleep. It stopped when she blew her nose. The hearing decreased in her right ear. The patient was stressed, could not sleep, and developed tension headaches. 
Otologic examination using a microscope revealed a moving tympanic membrane, especially in the anterior quadrant. This movement was synchronous with the patient's heart rate. Auscultation around the ear did not reveal a bruit and neurological examination was normal. Tuning fork examination revealed lateralization to the right ear on Weber's test examination and bone conduction was greater than air conduction in the right ear on Rinne's test. Rinne's test on the left ear was normal. Pure-tone audiography showed a right conductive hearing loss with an airbone gap of $10 \mathrm{~dB}$ and a normal tympanogram.

A CT scan of the brain and temporal bone revealed pneumocephalus in the right temporoparietal region that was continuous with the adjacent mastoid air cells, and a transverse fracture through the mastoid process. The craniotomy site in right temporal bone was noted (Figure 1A).

A meticulous mastoidectomy with unroofing of the epitympanum was performed. The entire mastoid air cell system was removed. The epitympanum was drilled to expose the incudomalleolar joint. We could not identify the dural defect that the CT scan had shown, nor could we see the fracture line. Widening of the aditus ad antrum was performed. The mastoid cavity was closed without fat obliteration.

After the operation, the pulsatile tinnitus ceased completely. The patient was very satisfied with the result. Otoscopic examination was normal with no evidence of pulsation.

A follow up CT scan at 3 months postoperatively demonstrated the absence of pneumocephalus and opacification of the right mastoid (Figure 1B). The audiogram showed normal hearing. The patient was still free from this symptom at 4-year follow-up visit.

\section{Discussion}

The causes of pulsatile tinnitus reported in the literature include arterial lesions, venous lesions, skull base/temporal bone/petrous lesions, and others causes, such as intracranial hypertension, anemia, palatal myoclonus, and a patulous Eustachian tube [1].

Otogenic pneumocephalus is an uncommon entity usually caused by otologic surgery, trauma, tumor or infection [2], though sometimes it can occur spontaneously [3, 4]. Temporal bone fracture is the most common cause of otogenic pneumocephalus $[5,6]$. This patient had a transverse fracture of the temporal bone and pneumocephalus in the subdural space in the right temporoparietal region continuous with the mastoid air cell system.

The most common presenting symptom of otogenic pneumocephalus is headache. Because of the lack of specific symptoms, pneumocephalus is usually not suspected and the diagnosis is made only after radiographic evaluation [6]. There are only two reported cases of pneumocephalus causing pulsatile tinnitus and both of them occurred after craniotomy and accidental opening of the mastoid air cells [7, 8].

This patient presented with pulsatile tinnitus synchronous with the moving tympanic membrane and heart beats. The source of this symptom was transmission of cerebrospinal fluid pulsation through the bony defect, which was transmitted to the mastoid air cell system that connects to the middle ear cavity. This caused the tympanic membrane to move in and out synchronous with pulse. This probably followed the fracture of the temporal bone, because air from the mastoid process entered the cranial cavity after the craniotomy for subdural hematoma removal during which there was accidental opening of the mastoid air cells.
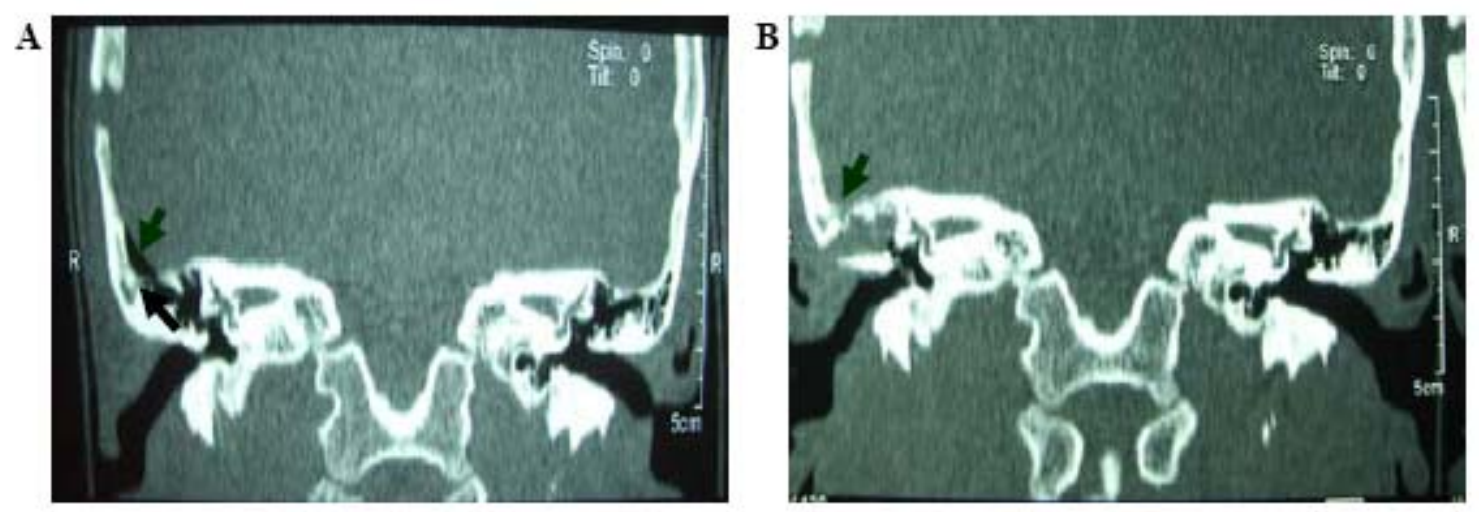

Figure 1. Coronal CT of the temporal bone. A: Preoperation showing pneumocephalus in the right temporoparietal region that was continuous with the adjacent mastoid air cells, and a transverse fracture through the mastoid process, B: Postoperation: absence of pneumocephalus 
The treatment of otogenic pneumocephalus depends on the degree of tension, symptomatology, and underlying cause. When associated with trauma or surgery, bed rest and close monitoring may suffice, although needle aspiration or re-exploration may be needed [6]. Treatment of pneumocephalus causing pulsatile tinnitus depends on the dural defect and includes craniotomy and closure of the fistula using a muscle fragment [7], and simple mastoidectomy and fat obliteration of the mastoid [8].

In this patient, mastoidectomy with unroofing the epitympanum without fat obliteration proved to be effective. Both the symptom and pneumocephalus completely resolved following the procedure. Our aim was to disconnect the space between middle ear/ mastoid system and the pneumocephalus, but we could not identify the bony defect at the base of the skull. We therefore only performed a mastoidectomy with unroofing of the epitympanum and widening of the aditus ad antrum. We did not obliterate the mastoid cavity, nor did we leave any packing in situ. We hoped that the pneumocephalus would resolve by escape of the air from cranial cavity through the enlarged space comprising the mastoid and middle ear cavity and via the Eustachian tube. We believe that the mechanism of pulsatile tinnitus in this case was transmission of the movement of the tympanic membrane to the ossicles and inner ear. The cause of the movement of the tympanic membrane was the transmission of brain pulsations through the small middle ear space and its pressure on the drum. After the surgery, the larger space of middle ear, aditus ad antrum and mastoid cavity may have been sufficient to relieve the pressure from brain pulsations, together with ventilation through the Eustachian tube. This is the hypothesis that we propose to explain the elimination of the symptom of pulsatile tinnitus of this patient.

There is one case report describing pulsatile tinnitus of venous origin, which was cured by mastoidectomy, with a 2-year follow-up [9]. We conclude that mastoidectomy and unroofing the epitympanum without fat obliteration is a very simple and effective option for the treatment of pulsatile tinnitus because of pneumocephalus.

\section{Acknowledgments}

I would like to express my sincere thanks to “associated Professor Somchart Sangsa-Ard” for his supervision, guidance and helping me in management of this patient.

I would like to give my special thanks to Professor Robert Peter Mills for assistance with the Englishlanguage presentation of the manuscript. The authors wish to declare no conflict of interest.

\section{References}

1. Waldvogel D, Mattle HP, Sturzenegger M, Schroth G. Pulsatile tinnitus-a review of 84 patients. J Neurol. 1998; 245:137-42.

2. Glynn F, Skinner LJ, Daly CA, Donnelly M. Otogenic pneumocephalus: case report and review of the literature. Rev Laryngol Otol Rhinol (Bord). 2007; 128: 59-61.

3. Wilkinson EP, Meyer TA, Rubinstein JT. Spontaneous otogenic pneumocephalus managed with the middle fossa approach. Acta Otolaryngol. 2007; 127:892-6.

4. Krayenbuhl N, Alkadhi H, Jung HH, Yonekawa Y. Spontaneous otogenic intracerebral pneumocephalus: case report and review of the literature. Eur Arch Otorhinolaryngol. 2005; 262:135-8.

5. Lefantzis D, Triantos S, Vontetsianos H, Dokianakis G. An unusual case of otogenic pneumocephalus. J Laryngol Otol. 1998; 112:1179-80.

6. Andrews JC, Canalis RF. Otogenic pneumocephalus. Laryngoscope. 1986; 96:521-8.

7. Saitoh Y, Takeda N, Yagi R, Oshima K, Kubo T, Yoshimine T. Pneumocephalus causing pulsatile tinnitus. Case illustration. J Neurosurg. 2000; 92:505.

8. Xenellis J, Nikolopoulos TP, Felekis D, Tzangaroulakis A. Pulsatile tinnitus: a review of the literature and an unusual case of iatrogenic pneumocephalus causing pulsatile tinnitus. Otol Neurotol. 2005; 26:1149-51.

9. Duvillard C, Ballester M, Redon E, Romanet P. Pulsatile tinnitus cured by mastoidectomy. Ann Otol Rhinol Laryngol. 2004; 113:730-3. 